\title{
A Case of Subcutaneous Hemangioma Presenting as a Preauricular Sinus
}

\author{
Sang-Gyun Jin, Min-Ju Kim, Jung-Mi Park and Kyoung-Ho Park \\ Department of Otolaryngology-Head \& Neck Surgery, The Catholic University of Korea College of Medicine, Seoul, Korea
}

$\begin{array}{ll}\text { Received } & \text { March 8, 2013 } \\ \text { Revised } & \text { March 29, 2013 } \\ \text { Accepted } & \text { April 1, 2013 }\end{array}$

Address for correspondence

Kyoung-Ho Park, MD, PhD

Department of Otolaryngology-

Head \& Neck Surgery,

The Catholic University of Korea

College of Medicine,

222 Banpo-daero, Seocho-gu,

Seoul 137-701, Korea

Tel $+82-2-2258-6213$

Fax +82-2-595-1354

E-mail khpent@catholic.ac.kr
Hemangioma is one of the most common benign neoplasm involved on head and neck. However, cavernous hemangiomas are relatively rare in preauricular sinus space and has rarely been reported around the world. Recently, a 44-year-old female patient came to our clinic with cavernous hemangioma involving the preauricular sinus. This case was surgically managed via excision. Preoperatively, it was difficult to differentiate the mass of hemangioma clinically and diagnostically from a preauricular fistula, a salivary gland tumor, or an enchondroma. This case report describes the subcutaneous hemangioma on a preauricular sinus, which is rarely seen in Korea. The clinical presentation and management are discussed with a review of the literature.

Korean J Audiol 2013;17:32-34

\section{Introduction}

Hemangioma is the commonly found benign neoplasm involved on head and neck. ${ }^{1-3)}$ It is also one of the most common benign vasofomative neoplasms of mainly infancy and childhood. ${ }^{4-6)}$ According to the previous studies, hemangiomas are classified into capillary, cavernous, and miscellaneous forms like verrucous, venous, arteriovenous. ${ }^{1,26)}$ The majority of hemangioma in early childhood regressed spontaneously. ${ }^{7)}$ However, an appearance and spontaneous regression of hemangioma in adults is very rare, especially in prearicular sinus. ${ }^{8)}$ Therefore, clinically, haemangiomas in adult are strongly required to appropriate management including surgical excision. In the present case study, we report a rare and an unusual case of haemangioma of the preauricular sinus with a review of the literature.

\section{Case Report}

A 44-year-old female came to our department with a 5-month history of intermittent right preauricular swelling and febrile sensation. She denied having had hearing loss, aural fullness, otaglia, tinnitus, and painful sensation. Her medical, surgical and family history were unremarkable. Upon physical examination, an ovoid shaped mass was softly palpable on $1 \mathrm{~cm}$ anterior from the tragus of right ear. The other findings of the head and neck were not significant but particularly, there was no pit around the softly palpable mass. Also, there was no secretion and painful sensation with pressure. Computed tomography (CT) demonstrated a $0.9 \mathrm{~cm}$ sized soft tissue mass in anterolateral to the right external auditory canal, which enhanced with contrast. The isolated mass was ovoid shaped and well-marginated without evidence of bony erosion of outer ear (Fig. 1). Based on the physical examination and computed tomography, preauricular fistula, salivary gland tumor, and enchondroma were possible diagnosis.

Under general anaesthesia, the mass was smoothly excised via the preauricular approach. The standard technique for preauricular sinus excision was used with a careful elliptical incision anterior to the crus and tragus on the superior portion of the mass and dissection without epithelial remnants. The size of the surgical specimen was $1.0 \times 0.8 \mathrm{~cm}$, reddish and soft (Fig. 2). Pathologic evaluation revealed well circumscribed, exophytic mass, delineated by squamous epithelium and proliferative small vessels (Fig. 3). On the basis of clinical examination and histopathology, a diagnosis of capillary haemangi- 
oma was concluded. The patient visited our clinic after a week with normal healing. She was also reviewed 1 and 3 months after surgical excision without the leision recurred.

\section{Discussion}

Hemangioma is a benign vascular soft tissue tumor that is probably an abnormal development of embryonic vasculature. ${ }^{6,7)}$ According to previous studies, hemangiomas are located in not only trunk or extermities, but also head and neck region. However, hemangioma in preauricular sinus has been rarely reported in the literature. ${ }^{9,10)}$ From this case, we learned and experienced the occurrence of hemangioma in preauricula sinus. Before surgical excision, some neoplasms were con-
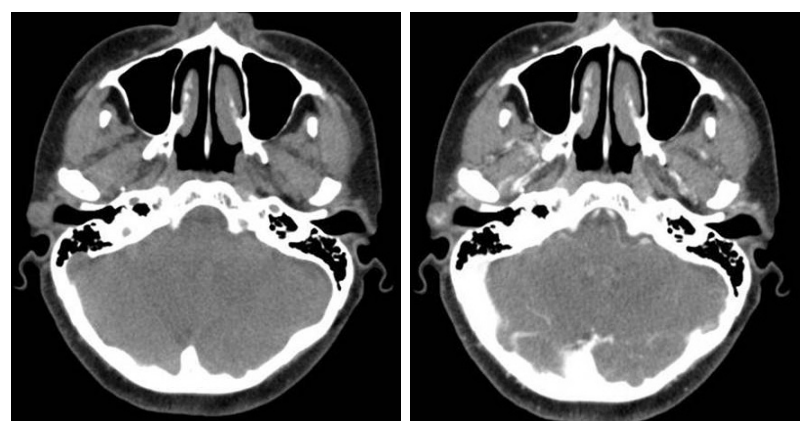

Fig. 1. Preoperative axial computed tomography demonstrates a soft tissue density in the right preauricular sinus. The middle ear cavity and mastoid antrum are clear.

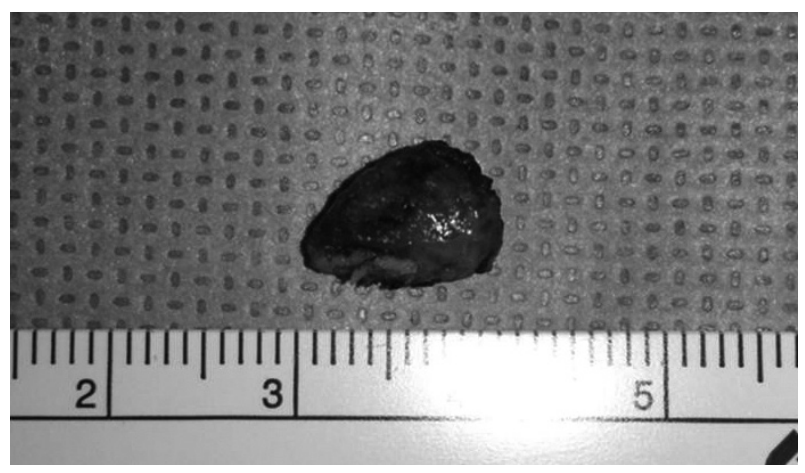

Fig. 2. The postoperative surgical specimen shows a red colored, smooth surfaced mass, measuring $1.2 \times 0.6 \mathrm{~cm}$. sidered including salivary gland tumor and enchondroma. The most common presenting symptoms and clinical signs of malignancy in auricle or parotid area are slowly growing palpable mass, which symptoms could be possibly painless, pain with tenderness, and repeated swelling with pain. Trismus, and facial paralysis would be possible clinical symptoms as well. Reviewing the literature, the peak incidece of benign and malignancy parotid gland tumor for Korean population are in the fifth decade for both males and females, and the eighth decades in males, third to fifth decades in females, respectively. ${ }^{11)}$ Considering the duration of symptoms, it varied from a few weeks to several years for both benign and malignancy. Preauricular fistula was also possibly considered because it arised on the preauricular sinus. However, no pit, which is typically found in preauricular sinus, was observed and then the pathological finding of the specimen showed the hemangioma arising in a preauricular sinus. If more diagnostic tools or needle aspiration for pathological findings were performed before surgical excision, more valuable diagnostic results would be obtained for more precised diagnosis.

Both contrast-enhanced CT and MR imaging are the useful diagnostic tools. Considering this case, MR imaging would be superior to CT because previous reported studies show that MR imaging clearly differentiated cavernous hemangiomas from hypervascular tumors. ${ }^{2)}$ Therefore, MR imaging would be a considerable diagnositc tool when evaluating a head and neck neoplasm, which could give more precise information to decide which treatments would give a better outcome.

According to the vessel type, three histopathological types of hemangioma have been reported. ${ }^{12)}$ A capillary type is characterized by prediminant capillary structures with proliferative activity that are invading to surrounding tissue, and a short clinical history. Cavernous type is characterized by large vessels with occasional mitotic activity and longer clinical history. Lastly, mixed type is characterized by having both the capillary and cavernous structures and similar clinical feature of cavernous type. $^{12)}$

Treatment options for hemangioma of head and neck area
Fig. 3. Histopathologic findings of the cavernous hemangioma was demonstrated. Low magnification shows well circumscribed, exophytic mass, delineated by squamous epithelium and vascular proliferation (H\&E stain, $\times 40$ ). High magnification shows mature capillary vessels lined by flattened endothelial cells and plump endothlial cell proliferation (H\&E stain $x$ 100).
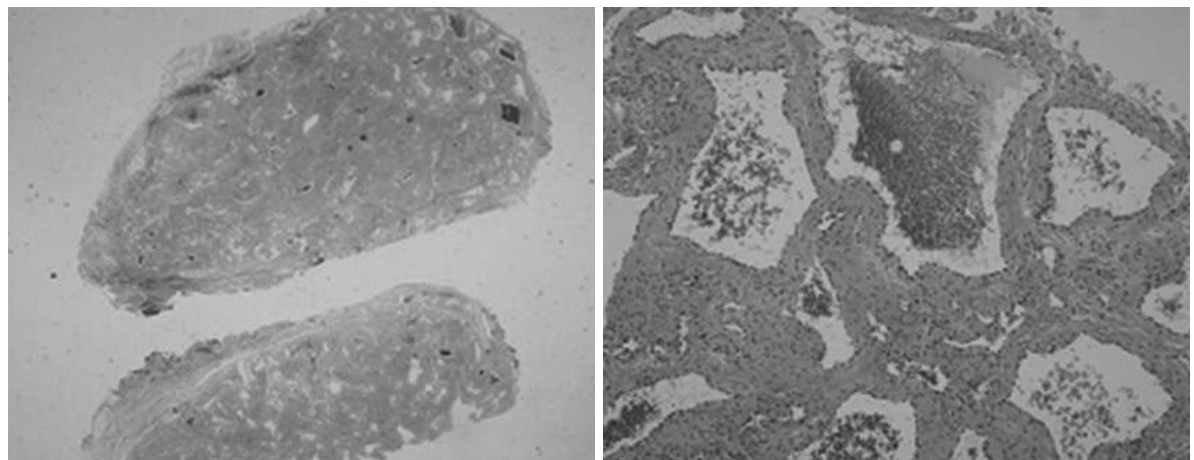
include irradiation, injection of sclerosing agents, embolization, and most effectively, surgical excision. ${ }^{4,6)}$ Careful surgical dissection is important to exclude malignancy and to prevent recurrence after surgery, which is caused by incomplete excision. Embolization and irradiation would be inadequate treatment because embolization alone is not effective if not followed by surgical excision, and irradiation has high complication potential, especially in children. ${ }^{8,9)}$ Furthermore, surgical dissection is recommended because hemangiomas are hardly spontaneously regressed and their size would be generally increased in adulthood. ${ }^{2)}$ In this case, therefore, surgical excision through a preauricular approach was the better treatment than other options. Furthermore, no complications or recurrence have been observed yet.

Hemangiomas are benign vascular tumors that are very rarely seen and reported in a preauricular sinus. From this single case report, the authors emphasize that radiologic findings would be generally insufficient for the correct diagnosis, and careful surgical excision is the treatment of choice.

\section{REFERENCES}

1) Cho KH, Kim KH, Lee KS, Yang HS. A case of capillary hemangioma in the external auditory canal. Korean J Otolaryngol-Head Neck Surg 2001;44:993-6.
2) Aspestrand F, Kolbenstvedt A. Vascular mass lesions and hypervascular tumors in the head and neck. Characteristics at CT, MR imaging and angiography. Acta Radiol 1995;36:136-41.

3) McMenamin M, Quinn A, Barry H, Sleeman D, Wilson G, Toner M. Cavernous hemangioma in the submandibular gland masquerading as sialadenitis: case report. Oral Surg Oral Med Oral Pathol Oral Radiol Endod 1997;84:146-8.

4) Capote A, Acero J, García-Recuero I, Rey J, Guerra B, de Paz V. Infratemporal-preauricular-cervical approach for resection of a cavernous intramasseteric hemangioma: a case report. J Oral Maxillofac Surg 2008;66:2393-7.

5) Martines F, Bentivegna D, Maira E, Marasà S, Ferrara S. Cavernous haemangioma of the external auditory canal: clinical case and review of the literature. Acta Otorhinolaryngol Ital 2012;32:54-7.

6) Scheinfeld NS, Silverberg NB, Weinberg JM, Nozad V. The preauricular sinus: a review of its clinical presentation, treatment, and associations. Pediatr Dermatol 2004;21:191-6.

7) Bartlett JA, Riding KH, Salkeld LJ. Management of hemangiomas of the head and neck in children. J Otolaryngol 1988;17:111-20.

8) Broniatowski M. Intramuscular hemangiomas of the masseter and sternomastoid muscles. Ear Nose Throat J 1993;72:303-5.

9) Batsakis JG. Tumors of the head and neck: clinical and pathological considerations. 2nd ed. Baltimore (MD): Williams \& Wilkins;1979. p.294-6.

10) Allen PW, Enzinger FM. Hemangioma of skeletal muscle. An analysis of 89 cases. Cancer 1972;29:8-22.

11) Lee YM, Choi HJ, Kim JW, Kim JH. Parotid gland tumors in a Korean population. J Craniofac Surg 2012;23:e205-9.

12) Beham $A$, Fletcher $C D$. Intramuscular angioma: a clinicopathological analysis of 74 cases. Histopathology 1991;18:53-9. 